\title{
A simple pointview for Kadec-1/4 theorem in the complex case
}

\author{
Pierluigi Vellucci
}

Received: 1 November 2014 / Accepted: 1 December 2014 / Published online: 17 December 2014 C) The Author(s) 2014. This article is published with open access at Springerlink.com

\begin{abstract}
This paper contains a simple and different pointview from the literature, in the best of my knowledge, to generalize well known results by Kadec (in $\mathbb{R}$ ) and Duffin and Eachus (in $\mathbb{C}$ ), concerning Riesz bases. Main goal of the present work is to overcome, at least partially, the limitations exhibited in the paper of Duffin and Eachus and in the book of Young for the Riesz bases. A consequence of the main theorem and its corollary is that the constant $\frac{\log 2}{\pi}$ can be replaced by $1 / 4$ (for complex $\lambda_{n}$ ).
\end{abstract}

Keywords Kadec's 1/4-theorem · Riesz basis · Exponential bases

Mathematics Subject Classification $42 \mathrm{C} 05 \cdot 42 \mathrm{C} 15 \cdot 42 \mathrm{C} 30$

\section{Introduction}

It is known that exponential Riesz bases $\left\{e^{i \lambda_{n} t}\right\}$ (with $\lambda_{n} \in \mathbb{R}$ ) are stable in the sense that a small perturbation of a Riesz basis produces a Riesz basis; it is proved by Paley and Wiener ([5,9]). The proof of the Paley-Wiener theorem does not provide an explicit stability bound. The celebrated theorem by M. I. Kadec shows that $1 / 4$ is the stability bound for the exponential basis on $L^{2}[-\pi, \pi]$.

The proof of theorem, as reported in the Young's textbook [9], applies for sequences of real numbers. Even earlier, however, Duffin and Eachus [2] shows that the PaleyWiener criterion is satisfied whenever the sequences are complex and $\frac{\log 2}{\pi}$ is a stability

Communicated by Salvatore Rionero.

P. Vellucci $(\varangle)$

Dipartimento di Scienze di Base e Applicate per l'Ingegneria,

Via Antonio Scarpa 14/16, 00161 Roma, Italy

e-mail: pierluigi.vellucci@sbai.uniroma1.it 
bound. For Young (page 38): "Whether the constant $\frac{\log 2}{\pi}$ can be replaced by $1 / 4$ (for complex $\lambda_{n}$ ) remains an unsolved problem." With Theorem C and Theorem D on [2] they consider sets which are on the borderline of being near a given orthonormal set, while the last part of their paper gives a simple formula for constructing sets near a given orthonormal set. Afterward, Duffin and Eachus apply this result (Theorem D) to the sequence of functions $\left\{e^{i \lambda_{n} x}\right\}$, where $\left\{\lambda_{n}\right\}, n=0, \pm 1, \pm 2, \ldots$ is a sequence of complex constants satisfying $\left|\lambda_{n}-n\right| \leq L$ for some constant L. The Duffin and Eachus's approach is deeper and more general than one of Young; in fact their work speaks of orthonormal sets and not of basis. In their paper can be read the following: "The above results on the non-harmonic Fourier series are an extension of previous knowledge in two respects. In the first place, Paley and Wiener were forced to assume that $\left\{\lambda_{n}\right\}$ was a real sequence. Secondly, they obtained the value $1 / \pi^{2}$ where we have $\ln 2 / \pi$. The best value for $L$ is not known; however a theorem of Levinson gives an upper limit of $1 / 4$ ".

Theorem 1 seeks to overcome the limitations exhibited in the paper of Duffin and Eachus and in the book of Young for the Riesz basis, introducing a limitation on the imaginary part of $\lambda_{n}$. A consequence of Theorem 1 and its corollary, is that the constant $\frac{\log 2}{\pi}$ can be replaced by $1 / 4$ (for complex $\lambda_{n}$ ).

Lastly, an example that shows $1 / 4$ cannot be replaced by a larger constant for complex case, are given in the appendix. For the latest results on generalizations and extensions of Kadec's theorem see: $[1,3,6]$.

\section{A class of sequences that improves the estimation of Duffin and Eachus}

Theorem 1 If $\left\{\bar{\lambda}_{n}\right\}=\left\{\lambda_{n}+i \mu_{n}\right\}$ is a sequence of complex numbers for which

$$
\left|\lambda_{n}-n\right| \leqq L<\frac{1}{4}, \quad n=0, \pm 1, \pm 2, \ldots
$$

and

$$
\left|\mu_{n}\right| \leqq \tau(L)<\frac{1}{\pi} \ln \left(\frac{2}{2-\cos \pi L+\sin \pi L}\right), \quad n=0, \pm 1, \pm 2, \ldots
$$

then $\left\{e^{i \bar{\lambda}_{n} t}\right\}$ satisfies the Paley-Wiener criterion and so forms a Riesz basis for $L^{2}[-\pi, \pi]$.

Proof It is to be shown that $\left\|\sum_{n}^{+\infty} c_{n}\left(e^{i n t}-e^{i \bar{\lambda}_{n} t}\right)\right\|<1$ whenever $\sum_{n}\left|c_{n}\right|^{2} \leqq 1$. Write

$$
\begin{aligned}
e^{i n t}-e^{i \bar{\lambda}_{n} t} & =e^{i n t}\left(1-e^{i \delta_{n} t} e^{-\mu_{n} t}\right) \\
& =e^{i n t}\left[1-e^{-\mu_{n} t}+e^{-\mu_{n} t}\left(1-e^{i \delta_{n} t}\right)\right]
\end{aligned}
$$

where $\delta_{n}=\lambda_{n}-n$. This time again, the trick is to expand the function $1-e^{i \delta t}$ $(-\pi \leq t \leq \pi)$ in a Fourier series relative to the complete orthonormal system $\left\{1, \cos n t, \sin \left(n-\frac{1}{2}\right) t\right\}_{n=1}^{\infty}$ and then exploit the fact that $\left|\lambda_{n}-n\right|$ is not too large. 
Then the expansion of $1-e^{i \delta t}$ is the same as the previous theorem. Let $\left\{c_{n}\right\}$ be an arbitrary finite sequence of scalars such that $\sum\left|c_{n}\right|^{2} \leq 1$. By interchanging the order of summation, using triangle inequality and the notation introduced in the Kadec's theorem on [9], it shows

$$
\begin{aligned}
& \left\|\sum_{n}^{+\infty} c_{n} e^{i n t}\left[1-e^{-\mu_{n} t}+e^{-\mu_{n} t}\left(1-e^{i \delta_{n} t}\right)\right]\right\| \\
& \leq \sup _{n}\left|1-e^{-\mu_{n} t}\right|\left\|\sum_{n}^{+\infty} c_{n} e^{i n t}\right\|+\sup _{n}\left(e^{-\mu_{n} t}\right)(A+B+C)
\end{aligned}
$$

From the assumptions of the theorem it is easily seen that $\sup _{n}\left(e^{-\mu_{n} t}\right) \leq e^{\tau \pi}$ and $\sup _{n}\left|1-e^{-\mu_{n} t}\right| \leq e^{\tau \pi}-1$ where $\tau=\tau(L)$. Now by some estimates on fraction expansions proved in [3], it has that

$$
\left\|\sum_{n}^{+\infty} c_{n}\left(e^{i n t}-e^{i \bar{\lambda}_{n} t}\right)\right\| \leq e^{|M|}-1+e^{|M|}(1-\cos \pi L+\sin \pi L)=: \lambda
$$

It is observed that with arbitrary $L<1 / 4$ and

$$
\tau(L)<\frac{1}{\pi} \ln \left(\frac{2}{2-\cos \pi L+\sin \pi L}\right)
$$

is obtained $\lambda<1$.

The following result shows that, in the hypotheses of the Theorem 1, it has $\left\{e^{i \bar{\lambda}_{n} t}\right\}$ satisfies the Paley-Wiener criterion for $\left|\bar{\lambda}_{n}-n\right|<1 / 4$ even when $\left\{\bar{\lambda}_{n}\right\}$ is a complex sequence.

Corollary 1 For each $L<\frac{1}{4}$, one has

$$
\text { (i) }\left|\mu_{n}\right| \leq \frac{\ln 2}{\pi} ; \quad \text { (ii) }\left|\bar{\lambda}_{n}-n\right| \leq \frac{1}{4}
$$

Proof The proof of first relation (i) is trivial and is left to the reader. Noting that

$$
\left|\bar{\lambda}_{n}-n\right| \leq\left|\lambda_{n}-n\right|+\left|\mu_{n}\right| \leq L+\frac{1}{\pi} \ln \left(\frac{2}{2-\cos \pi L+\sin \pi L}\right)
$$

relation (ii) is verified if $\bar{x}-\ln \left(1+\frac{\sin \bar{x}-\cos \bar{x}}{2}\right) \leq \frac{\pi}{4}$ with $\bar{x}=\pi L$. Let us consider the function $f(\bar{x})$, defined as follow:

$$
f(\bar{x})=\bar{x}-\ln \left(1+\frac{\sin \bar{x}-\cos \bar{x}}{2}\right)
$$


It comes to prove that the function $f(\bar{x})-\bar{x}:=g(\bar{x})$ is convex. Rewrite the function $g(\bar{x})$ using the relationship $(\sin \bar{x}-\cos \bar{x}) / 2=\frac{\sqrt{2}}{2} \sin \left(\bar{x}-\frac{\pi}{4}\right)$ and so $g(x)=-\ln \left(1+\frac{\sqrt{2}}{2} \sin x\right)$ for $x=\bar{x}-\pi / 4$. Bearing in mind that a function is convex if and only if it is midpoint convex, it must be demonstrated that $2 g\left(\frac{x+y}{2}\right) \leq g(x)+g(y)$, and hence

$$
-2 \ln \left(1+\frac{\sqrt{2}}{2} \sin \frac{x+y}{2}\right) \leq-\ln \left(1+\frac{\sqrt{2}}{2} \sin x\right)-\ln \left(1+\frac{\sqrt{2}}{2} \sin y\right)
$$

where $y=\bar{y}-\pi / 4$. From properties of logarithms and by applying Prosthaphaeresis formulas, Werner formulas, and half-angle formulae, it has

$$
\sqrt{2} \sin \left(-\frac{x+y}{2}\right) \leq \cos ^{2} \frac{x-y}{4}
$$

Rewriting $-\frac{x+y}{2}=\frac{\pi}{4}-\frac{\bar{x}-\bar{y}}{2}-\bar{y} \leq \frac{\pi}{4}-t$ with $t=\frac{\bar{x}-\bar{y}}{2} \in[0, \pi / 4]$, it becomes $\sqrt{2} \sin \left(\frac{\pi}{4}-t\right) \leq \cos ^{2} \frac{t}{2}$, that is verified over $[0, \pi / 4]$. Then $f(x)$ is convex. Denoting with $P_{1}(0, \ln 2), P_{2}(\pi / 4, \pi / 4)$ two points belonging to graphic of $f(x)$ and from an obvious properties of convex functions: $f(x) \leq \frac{\pi-\ln 16}{\pi} x+\ln 2$ (the straight line for $P_{1}, P_{2}$ ), by the right side term that is less than $\frac{\pi}{4}$ if $x \leq \frac{\pi}{4}$, it is concluded the claim.

\section{Conclusions}

Theorem 1 and Corollary 1 responding to the outstanding questions of Duffin, Eachus and Young, essentially because this paper shows that the constant $\frac{\log 2}{\pi}$ can be replaced by $1 / 4$, also for the complex case. Moreover, from Corollary 1 , it has $\left\{e^{i \bar{\lambda}_{n} t}\right\}$ satisfies the Paley-Wiener criterion for $\left|\bar{\lambda}_{n}-n\right|<1 / 4$ even when $\left\{\bar{\lambda}_{n}\right\}$ is a complex sequence. Two lemmas present in appendix (an extension to complex case of result present on [9]) prove that Kadec's 1/4-theorem is "best possible": the system $\left\{e^{i \bar{\lambda}_{n} t}\right\}$ constitutes a basis for $L^{2}[-\pi, \pi]$ whenever every $\bar{\lambda}_{n}$ is complex and $\left|\lambda_{n}-n\right| \leqq L,\left|\mu_{n}\right| \leqq \tau(L)$ but not constitute a basis when $L=1 / 4$. Equally interesting is the fact that $\tau(L)$ is not specified in the proofs of Lemmas 1 and 2 and, into this proofs, it is not necessary that it assumes the logarithmic expression (2).

In Duffin and Eachus [2] one reads: "It is a curious parallelism that $\log 2 / \pi$ and $1 / 4$ are in the same ratio as the limits of Takenaka and Schoenberg in a somewhat similar unsolved problem". See: [7,8]. In [8] is reported a particular case of one of Takenaka's theorems [7]: "If every derivative of an integral function $f(z)$ has a zero inside or on the unit circle and if $\lim _{\sup _{r \rightarrow \infty}} \frac{\log M(r)}{r}<\log 2$ then $f(z)$ is a costant". $[M(r)$ is the maximum modulus in $|z| \leq r$ of function]. The author write that this condition is probably not "best possible": $\sin \frac{\pi}{4} z-\cos \frac{\pi}{4} z$ shows that $\log 2$ cannot be replaced by any number larger than $\pi / 4$, and this may well be the true value. 
A possible development of the work would be compare proof of Kadec's-1/4 theorem (complex case) with question in [8].

Acknowledgments I would like to thank Prof. Antonio Avantaggiati for the precious discussions who helped me to write this paper.

Open Access This article is distributed under the terms of the Creative Commons Attribution License which permits any use, distribution, and reproduction in any medium, provided the original author(s) and the source are credited.

\section{Appendix: $L=1 / 4$ as best possible choice}

The two lemmas below follows by Young's book just adapting to complex case in this paper. For the theory of entire function and the proof of Lemma 1, see respectively on the chapter 2 and on pages and 103-105 of Young's book.

Lemma 1 If $\lambda_{n}=n+\varepsilon+i \tau(\varepsilon)(n=1,2,3 \ldots)$, where $\varepsilon>-1$, and

$$
H(z)=\prod_{n}\left(1-\frac{z^{2}}{\lambda_{n}^{2}}\right)
$$

then

$$
H^{\prime}\left(\lambda_{n}\right)=(-1)^{n} \Gamma^{2}(1+\varepsilon+i \tau(\varepsilon)) \frac{\Gamma(n)}{\Gamma(n+1+2 \varepsilon+2 i \tau(\varepsilon))}
$$

Now, using the thesis of this lemma, is shown the next result.

\section{Lemma 2 If}

$$
\lambda_{n}= \begin{cases}n+\varepsilon+i \tau(\varepsilon), & n>0 \\ 0, & n=0 \\ n-\varepsilon-i \tau(\varepsilon), & n<0\end{cases}
$$

then, for $\varepsilon \geq 1 / 4$, the system $\left\{e^{i \lambda_{n} t}\right\}$ is not a Riesz basis for $L^{2}[-\pi, \pi]$.

Proof Suppose it were. Then the system of reproducing functions $\left\{K_{n}(z)\right\}, K_{n}(z)=$ $\sin \pi\left(z-\lambda_{n}\right) / \pi\left(z-\lambda_{n}\right)$, would be a Riesz basis for Paley-Wiener space $P$, since the Fourier transform is an isometry. Put

$$
F_{n}(z)=\frac{F(z)}{F^{\prime}\left(\lambda_{n}\right)\left(z-\lambda_{n}\right)}
$$

where $F(z)=\prod_{n}\left(1-z^{2} / \lambda_{n}^{2}\right)$. Then $F_{n}\left(\lambda_{k}\right)=\delta_{n k}$, and $F_{n}$ belongs to P. Accordingly, $\left\{F_{n}(z)\right\}$ is biorthogonal to $\left\{K_{n}(z)\right\}$ in $\mathrm{P}$ and so must also be a Riesz basis for P. In particular, the series

$$
\sum_{n} c_{n} \frac{F(z)}{F^{\prime}\left(\lambda_{n}\right)\left(z-\lambda_{n}\right)}
$$


must converge in the topology of $\mathrm{P}$, and hence pointwise, whenever $\left\{c_{n}(z)\right\} \in L^{2}$. By the converse to Hölder's inequality, this can happen only if

$$
\sum_{n \neq 0}\left|\frac{1}{\lambda_{n} F^{\prime}\left(\lambda_{n}\right)}\right|^{2}<\infty
$$

But by Lemma (1),

$$
F^{\prime}\left(\lambda_{n}\right)=(-1)^{n} \Gamma^{2}(1+\varepsilon+i \tau(\varepsilon)) \frac{\Gamma(n)}{\Gamma(n+1+2 \varepsilon+2 i \tau(\varepsilon))}
$$

and Stirling's formula,

$$
\frac{\Gamma(n)}{\Gamma(n+a)} \sim e^{-a \ln n}
$$

shows that

$$
\sum_{n \neq 0}\left|\frac{1}{\lambda_{n} F^{\prime}\left(\lambda_{n}\right)}\right|^{2}=\infty
$$

for $\varepsilon \geq 1 / 4$ and the contradiction proves the lemma.

\section{References}

1. Christensen, O.: Frames, Riesz bases and discrete gabor/wavelet expansions. Am. Math. Soc. 38(3), 273-291 (2001)

2. Duffin, R.J., Eachus, J.J.: Some notes on an expansion theorem of Paley and Wiener. Bull. Am. Math. Soc. 48, 850-855 (1942)

3. Markushevich, A.I.: Theory of Functions of a Complex Variable, vol. 2. Prentice-Hall, Englewood Cliffs (1965)

4. Nakamura, A.: Basis properties and complemets of complex exponential systems. Hokkaido Math. J. 36, 193-206 (2007)

5. Paley, R., Wiener, N.: Fourier transforms in the complex domain. American Mathematical Society. Colloquium Publications, New York (1934)

6. Sun, W., Chui, X.C.K.: On Kadec's 1/4-theorem and the stability of gabor frames. Appl. Comput. Harmon. Anal. 7(2), 239-242 (1999)

7. Takenaka, S.: On the expansion of integral transcendental functions in generalized Taylor's series. Proc. Phys. Math. Soc. Jpn. 14, 529-542 (1932)

8. Whittaker, J.M.: Interpolatory Function Theory. Cambridge, London (1935)

9. Young, R.M.: An introduction to nonharmonic Fourier series. Academic Press, New York (2001) 